\title{
PSFC/JA-97-25
}

\section{Volume Recombination and Opacity in Alcator C-Mod Diverter Plasmas}

\author{
J.L. Terry, B. Lipschultz, A.Yu. Pigarov ${ }^{1,2}$,
} S.I. Krasheninnikov ${ }^{1}$, B. LaBombard, D. Lumma, H. Ohkawa, D. Pappas, M. Umansky

December 1997

Plasma Science and Fusion Center Massachusetts Institute of Technology

Cambridge, MA 02139

${ }^{1}$ Also at I.V. Kurchatov Institute of Atomic Energy, Moscow, Russian Federation.

${ }^{2}$ Also at The College of William and Mary, Williamsburg, VA, USA.

To be published in Physics of Plasmas.

This work was supported in part by the U. S. Department of Energy Contract No. DEAC02-78ET51013. Reproduction, translation, publication, use and disposal, in whole or in part by or for the United States government is permitted. 


\title{
Volume Recombination and Opacity in Alcator C-Mod Divertor Plasmas
}

\author{
J.L. Terry, B. Lipschultz, A.Yu. Pigarov ${ }^{+, *}$, S.I. Krasheninnikov ${ }^{+}$, B. LaBombard, \\ D. Lumma, H. Ohkawa, D. Pappas, and M. Umansky
}

Plasma Science and Fusion Center, Massachusetts Institute of Technology, Cambridge, Massachusetts, 02139, USA

\begin{abstract}
Volume recombination within the Alcator C-Mod [I.H. Hutchinson et al., Phys. Plasmas 11511 (1994)] divertor plasma is measured and found to be a significant fraction of the total ion sink under detached divertor conditions. The recombination occurs in regions where $\mathrm{T}_{e} \sim 1 \mathrm{eV}$ and $\mathrm{n}_{e} \sim 10^{21} \mathrm{~m}^{-3}$. Measurements of the spatial distribution of the recombination are presented. The determinations of the recombination rates are made by measuring the $\mathrm{D}^{0}$ Balmer spectrum and by using a collisional radiative model describing the level populations, ionization and recombination of $\mathrm{D}^{0}$. The concept of 'recombinations per Balmer series photon' is developed to simplify the determinations. Measurements of the opacity of $\mathrm{Ly}_{\beta}$ emission are presented. It is observed that up to $50 \%$ of the $\mathrm{Ly}_{\beta}$ emission is trapped, indicating that $\mathrm{Ly}_{\alpha}$ is strongly trapped in some cases. The effects of $\mathrm{Ly}_{\alpha, \beta}$ trapping on the 'recombinations per photon' curves are calculated and considered in the recombination rate determinations. Observations indicating the presence of Molecular Activated Recombination are discussed.
\end{abstract}

+ Also at I.V. Kurchatov Institute of Atomic Energy, Moscow, Russian Federation

* Also at The College of William and Mary, Williamsburg, VA, USA

PACS numbers: 34.80.Lx, 52.25.Rv, 52.52.Qt, 52.25.Ya

PSFC Report \# PSFC/JA-97-25, to be published in Phys. Plasmas 


\section{INTRODUCTION}

Stimulated by the experimental observation of very low $(\lesssim 3 \mathrm{eV})$ temperatures in the divertors of tokamaks[1,2], there has been much interest in studying experimentally the recombination of the plasma ions and electrons within the magnetically confined plasma. Typically this phenomenon is called 'volume recombination', to distinguish it from recombination which occurs at the material surfaces. In a very general sense, if volume recombination fully removed the ion flux to divertor plates, a condition of complete insulation of plasma from the material walls of its containment vessel would have been achieved. Such a condition is significant, since it means that a multi-keV plasma has been effectively insulated from the walls which surround it, and its enormous heat efflux has been distributed more uniformly on the surfaces via radiation. In a more specific sense, the 'insulation' provided by volume recombination results in a reduced ion flux to the divertor plates, which is accompanied by an even greater reduction in energy flux. The energy carried by a neutral to the plate is $\sim 2 \mathrm{~T}_{i}$, while for an ion it is $\sim 5 \mathrm{~T}_{i}$ plus the $13.6 \mathrm{eV}$ of potential energy implicit in an ion-electron pair. This difference reduces both the target heat load and physical sputtering.

Low divertor-plasma temperatures are achieved under 'detached' [3,4] and/or highly radiating conditions. Modelling has shown that recombination should be significant in these divertor plasmas, and that it is potentially responsible for removing a large fraction of the ion flux [4-9]. The first experimental measurements of significant volume recombination in a tokamak divertor were made on Alcator C-Mod[10,11] where its quantitative signature was found in the emission spectrum of $\mathrm{D}^{\circ}$, and where the ion sink due to recombination was found to be comparable to the ion loss to the plates. Experimental spectroscopic evidence for recombination in the divertor plasmas of ASDEX-Upgrade[12], DIII-D[13], and JET[14] now exists as well. Measurements on linear devices[15,16] have also shown the importance of recombination in simulations of plasma detachment. However, considerably more detail is required of the observations, and more quantitative comparisons with models are required before the models can be used to predict the conditions which might exist in a dissipative divertor of a fusion reactor. In particular, of the many questions still to be answered about the various phenomena occurring in a divertor, it is important to determine: 
1. the scaling and spatial distribution of the recombination,

2. the importance of the trapping of $\mathrm{Ly}_{\alpha, \beta}$ line emission on recombination,

3. the importance of Molecular Activated Recombination (MAR) $[17-20,16]$.

It is the purpose of this paper to address these issues by studying the divertor plasma of the Alcator C-Mod Tokamak. Alcator C-Mod can produce high density $\left(\mathrm{n}_{e}^{\text {divertor }} \gtrsim 10^{21} \mathrm{~m}^{-3}\right.$ ), detached discharges with reactor-like Scrape-Off-Layer (SOL) power flows $\left(\mathrm{q}_{\|} \lesssim 500 \mathrm{MW} / \mathrm{m}^{2}\right)$, and thus provides an ideal experiment for the study of these phenomena. This paper will also present an analysis technique which simplifies the evaluation of the total 3-body and radiative recombination rate using the intensities of the Balmer series lines. This technique relates the number of recombinations to the number of photons within a given Balmer series line for a recombining plasma.

\section{CONCEPTUAL APPROACH AND EXPERIMENTAL DETAILS}

Recombination of the majority ion species in a tokamak divertor can occur in three ways:

1. radiative recombination $\mathrm{D}^{+}+\mathrm{e} \rightarrow \mathrm{D}^{0}+\mathrm{h} \nu$,

2. 3-body recombination: $\mathrm{D}^{+}+\mathrm{e}+\mathrm{e} \rightarrow \mathrm{D}^{\mathrm{O} *}+\mathrm{e}$, and

3. Molecular Activated Recombination (MAR) [17-20], whose multiple pathways require the presence of vibrationally excited hydrogenic molecules, $\mathrm{D}_{2}(\nu)$, i.e.

- $\mathrm{D}_{2}(\nu)+\mathrm{e} \rightarrow \mathrm{D}^{0}+\mathrm{D}^{-}$, followed by $\mathrm{D}^{-}+\mathrm{D}^{+} \rightarrow \mathrm{D}^{0}+\mathrm{D}^{0 *}$, and

- $\mathrm{D}_{2}(\nu)+\mathrm{D}^{+} \rightarrow \mathrm{D}_{2}^{+}+\mathrm{D}^{0}$, followed by $\mathrm{D}_{2}^{+}+\mathrm{e} \rightarrow \mathrm{D}^{0}+\mathrm{D}^{0 *}$,

where $D^{0 *}$ means that the atom is typically in an excited state.

In today's medium- to high-density tokamaks, the first process, although important for diagnostic purposes, is the primary recombination pathway only at temperatures $\gtrsim 4 \mathrm{eV}$ when ionization, not recombination, will dominate. The latter two processes are predicted to be important at temperatures below $\sim 2 \mathrm{eV}$ and in the 1-3 $\mathrm{eV}$ range, respectively. Both 3-body recombination and MAR typically leave a recombined atom in an excited state, which can subsequently either decay to the ground 
state or re-ionize. In most of the cases of relevance to tokamaks, the decay to the ground state will occur by photon emission. For this reason, the study of recombination is possible through the spectroscopy of hydrogenic line emission. It also calls for a distinction between those recombinations which end with an atom in the ground state, something we will call a 'complete' recombination, and those which re-ionize. Only 'complete' recombinations will be significant in the ionization/recombination balance in tokamaks.

For the determination of the 'complete' recombination rates in Alcator C-Mod, measurements of the Balmer and Lyman series lines of $\mathrm{D}^{0}$ are used. Because the Balmer lines occur in the visible and the Lyman lines appear in the vacuumultraviolet, the spatial coverage of Balmer series measurements is much more extensive. The cross-section of the Alcator C-Mod device, the magnetic reconstruction of a typical diverted, lower single-null discharge, and the different diagnostic views used in this study are shown in Fig. 1. The visible measurements are made using a spectrometer which records spectra from multiple fibers, each with a different view of the plasma. Additional $\mathrm{D}_{\alpha}$ measurements are made using two interference-filter-based diode arrays[21]. A description of the visible spectrometer and the calibrations can be found in Ref. [10]. The Lyman series measurements were made with a grazing incidence spectrometer with a spectral resolution of $\sim .18 \mathrm{~nm}$ at $100 \mathrm{~nm}$. For this work its line-of-sight passed just over the $\mathrm{X}$-point and viewed the inner divertor above the inner strike point, the 'inner nose view' as shown in Fig. 1. Another visible view has been aligned to view along the grazing incidence instrument's sight line and to have nearly the same footprint[22].

The discharges examined in this report had plasma currents of 0.8 or 1.0 MA, $\bar{n}_{e} \lesssim 3 \cdot 10^{20} \mathrm{~m}^{-3}, P_{I C R F} \leq 1.6 \mathrm{MW}$ and both L- and $\mathrm{H}$-mode energy confinement. (A description of the general machine operation can be found elsewhere [23].) It is an experimental observation that the inner divertor plate is almost always in a detached state as soon as C-Mod discharges are diverted. At high densities and/or low SOL power flows, the outer divertor also detaches[24]. This transition is characterized by a large drop in ion current, plasma pressure, and electron temperatures as measured by probes in the plates[1]. After detachment the temperatures measured by the plate 


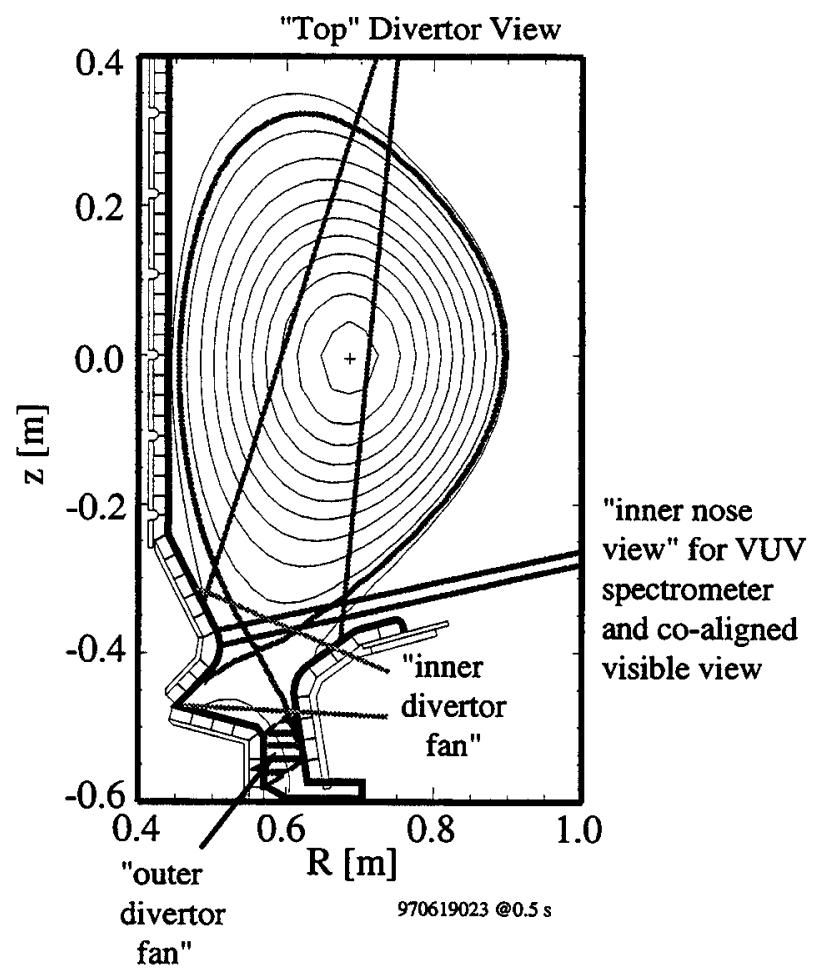

Figure 1: A cross section of Alcator C-Mod, showing the equilibrium reconstruction of the magnetic surfaces, the closed divertor, and the various views used in this study. Spatially resolved $D_{\alpha}$ measurements are made within the "top view" and "inner divertor fan". Multiple chords within the "inner" and "outer divertor fans" have a spatial resolution of $\sim 1 \mathrm{~cm}$. A view used for Lyman series (VUV) measurements, together with an almost identical visible view, is also shown. Probes embedded in the divertor plates are used to measure the ion current to the plates. 
probes are $\sim 1 \mathrm{eV}$. See Ref. [24] and references therein for detachment studies on Alcator C-Mod.

\section{ANALYSIS TECHNIQUES}

A visible spectrum typical of those measured along the "inner nose view", ending at the inner detached divertor above the strike point, is shown in Fig. 2. Balmer lines from $n=6,7,8 \ldots 11 \rightarrow 2$ are evident. These lines are strongly Stark broadened, indicative in this case of an electron density of $1.0-1.2 \times 10^{21} \mathrm{~m}^{-3}$. There is also a change in the continuum level between $\lambda \lesssim 370 \mathrm{~nm}$ and $\lambda \gtrsim 395 \mathrm{~nm}$. The very high- $n$ lines merge into the brighter continuum.

A radiative recombination edge in an isolated $\mathrm{D}^{0}$ atom occurs at $\lambda_{\text {edge }}=364.5 \mathrm{~nm}$. The extension of the higher intensity continuum to $\lambda \geq \lambda_{\text {edge }}$ and the merging of the discrete lines into the continuum are a result of the high electron density in the atoms' environment. The high $\mathrm{n}_{e}$ produces statistical plasma microfields which move very high- $n$, otherwise-bound states into the continuum. It also causes Stark broadening and causes the recombination continuum to extend to wavelengths above the 'isolated' atom edge. These effects have been incorporated into a collisional-radiative (CR) code describing the atomic and continuum emission in plasmas, CRAMD[20], and spectra like those shown in Fig. 2 have been simulated in detail by the code[25].

The analyses of this spectrum and others like it including the $\mathrm{D}_{\alpha, \gamma}$ lines, as well as analyses of the Lyman series, follow the techniques detailed in Refs. [10,11]. They will only be summarized here, in order to provide the background for extension to the simpler concept of 'recombinations per photon' and to evaluate recombination rates in those cases which are not optically thin.

As mentioned above, 3-body 'complete' recombination occurs dominantly by recombination into excited states which decay radiatively to the ground state. Thus if 3 -body recombination is the only process populating the atomic excited states, measurement of the total number of radiative decays to the ground state per unit time is a direct measure of $\approx 90 \%$ of the total recombination rate (excluding MAR)[10]. (The last $\approx 10 \%$ of the 'complete' recombinations occur by collisional de-excitation and by radiative recombination directly into the ground state.) Of course, the experimental situation is complicated by the fact that the atomic excited states can also 


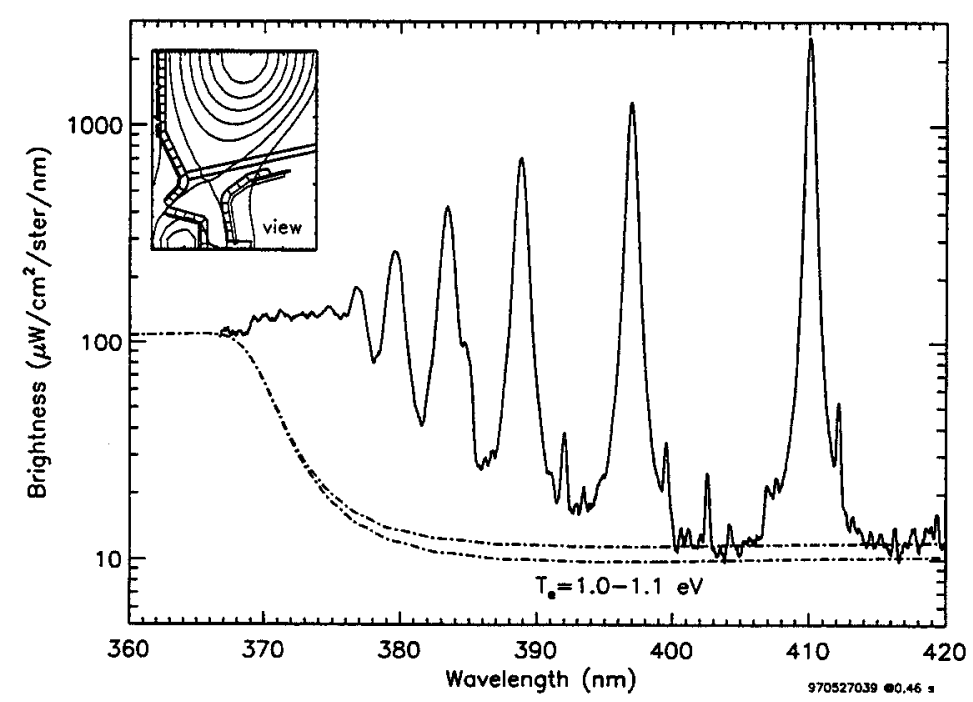

Figure 2: The higher-n $D^{0}$ Balmer series lines $(n=11,10, \ldots 6 \rightarrow 2)$. The widths of the Stark broadened lines correspond to a density of $\sim 1 \times 10^{21} \mathrm{~m}^{-3}$. Also shown is the radiative recombination continuum spectrum predicted for a plasma of $T_{e}=1.0$ and $1.1 \mathrm{eV}$ with $n_{e}=n_{i}=1.2 \times 10^{21} \mathrm{~m}^{-3}$ and $\Delta L=0.021 \mathrm{~m}$. The view is along the chord shown in the inset and ends at the inner detached divertor plate.

be populated by electron impact excitation from the ground state and/or self absorption of Lyman series lines. The contributions of excitation and recombination can be distinguished because they populate the excited levels with different scalings vs. $n$, the principal quantum number. Recombination tends to populate the high- $n$ levels (in a Saha-Boltzmann distribution), while the excitation distribution is very heavily weighted toward low $n$ levels. Finding those levels which are populated primarily by recombination is the crucial step enabling the determination of the 3-body (and radiative) recombination rate. The procedure may be summarized as:

- determine from the spectrum which excited levels of $\mathrm{D}^{0}$ are populated primarily by recombination,

- determine $\mathrm{n}_{e}$ from the Stark-broadened high- $n$ line(s),

- use the recombinations per photon curves at $\mathrm{T}_{e} \sim 1 \mathrm{eV}$ with the brightnesses of any or all of the 'recombination' lines to deduce the recombination rate within the field-of-view. 


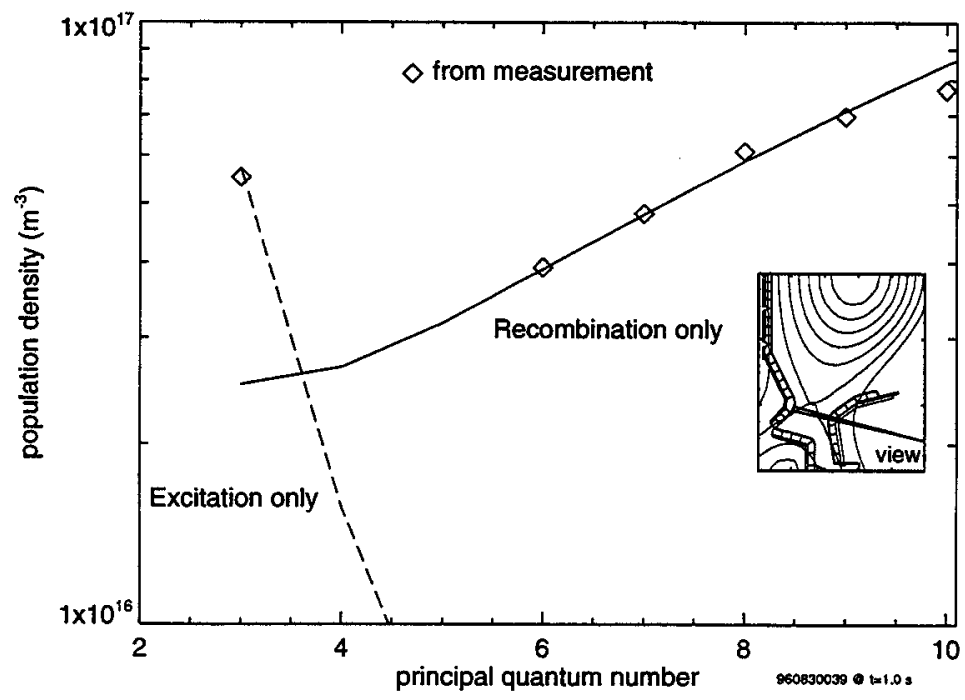

Figure 3: Comparison of the atomic level populations: The symbols are determined from a spectrum measured along a chord viewing the inner, detached divertor plate from within the "inner divertor fan". The theoretical prediction of the population densities resulting from recombination only is given by the solid line. The prediction for population densities resulting from ground state excitation is shown with the dashed line.

The strong difference in scaling with $n$ is shown in Fig. 3, where recombination and excitation population distributions are compared. Also shown are the population densities determined from measurement of the Balmer spectrum. In order to determine the population distribution in the experimental situation, a $\mathrm{D}^{0}$ spectrum like that shown in Fig. 2, containing as many Balmer series lines as possible, is analyzed. The line brightnesses are measured and related to the population densities by:

$$
N_{n}=\frac{B_{n \rightarrow 2} 4 \pi}{A_{n \rightarrow 2} \Delta L^{\text {cold }}}
$$

where the $\mathrm{A}_{n \rightarrow 2}$ 's are the spontaneous emission coefficients and $\Delta \mathrm{L}^{\text {cold }}$ is the length of the region giving rise to the emission as measured along the instrumental line-of-sight, the brightness is in units of photons/s/unit-area/ster, and the lines are assumed to be optically thin. $\Delta \mathrm{L}^{\text {cold }}$ is unknown a priori in the experiment, but is determined by finding the value which yields a good match between the $\mathrm{N}_{n \geq 6}$ 's and the population densities calculated for a plasma of $\mathrm{T}_{e}^{\text {cold }}$ and $\mathrm{n}_{e}^{\text {cold }}$. The density where the high- $n$ Balmer lines are emitted and where the 3 -body recombination occurs, $\mathrm{n}_{e}^{\text {cold }}$, is determined from the Stark broadening of the high- $n$ Balmer lines. $T_{e}^{\text {cold }}$ is less accurately 
determined from the change in the continuum emission around $\lambda_{\text {edge }}$, as shown in Fig. 2.

The population distributions shown in Fig. 3 are calculated assuming no radiation trapping using a CR model like that of Johnson and Hinnov[26], Fujimoto et al. [27], or CRAMD. (The levels $n \gtrsim 5$ will be close to Saha-Boltzmann equilibrium.) For the purposes of determining what lines in the series are populated primarily by recombination, nothing more accurate must be done, since the scalings are so different. Those lines (e.g. lines with $n_{\text {upper }} \geq 6$ in Fig. 3 ) are then used in the determination of the total recombination rate, which is done in conjunction with the $\mathrm{CR}$ model, since it predicts both the total recombination rate and the population densities for any given $\mathrm{T}_{e}$ and $\mathrm{n}_{e}$. The number of recombinations per photon of any desired line is thus determined. This is the recombination analog for what Johnson and Hinnov [26] have done in the ionizing case. The CR model in the recombining case shows a rather weak temperature and density dependence on the number of 'recombinations per photon' for some $\mathrm{D}^{0}$ lines. An examples of this quantity for $\mathrm{D}_{\gamma}$ vs $\mathrm{T}_{e}$ is shown as the top, solid line in Fig. 4 under the assumption that the Lyman series lines are optically thin. Here CRAMD has been used, although for this optically thin case the CR models of Ref. [27] or Ref. [26] give essentially the same results.

\section{REABSORPTION AND TRAPPING OF LYMAN LINE EMISSION}

Both the ionization and recombination rates are affected if the Lyman series photons do not escape the plasma. This is due to the fact that these photons can be the last stage in the process of 'complete' recombination which began by recombination into excited states. Since most of the 'complete' 3-body recombinations occur by emission of $\mathrm{Ly}_{\alpha}(\sim 60 \%)$ or $\mathrm{Ly}_{\beta}(\sim 10 \%)$, any reduction in these channels due to photon loss reduces the net recombination rate and increases the net ionization rate. After emission, photons can be absorbed by neutral atoms in their paths. The absorption mean-free-paths are easily calculated, and for $\mathrm{Ly}_{\alpha, \beta, \gamma}$ photons are $\lambda_{m f p}=0.0018 / \mathrm{N}_{0}^{20}$, $0.012 / \mathrm{N}_{0}^{20}$, and $0.035 / \mathrm{N}_{0}^{20}$ [in $\mathrm{m}$ ] respectively, for $1 \mathrm{eV}$ atoms, where $\mathrm{N}_{0}^{20}$ is the atomic density in units of $10^{20} \mathrm{~m}^{-3}[18]$. These absorbing, excited atoms can re-radiate or suffer ionizing collisions (as well as other collisions). In the first case a photon can continue to diffuse through the absorbers. However, in the second case a Lyman 


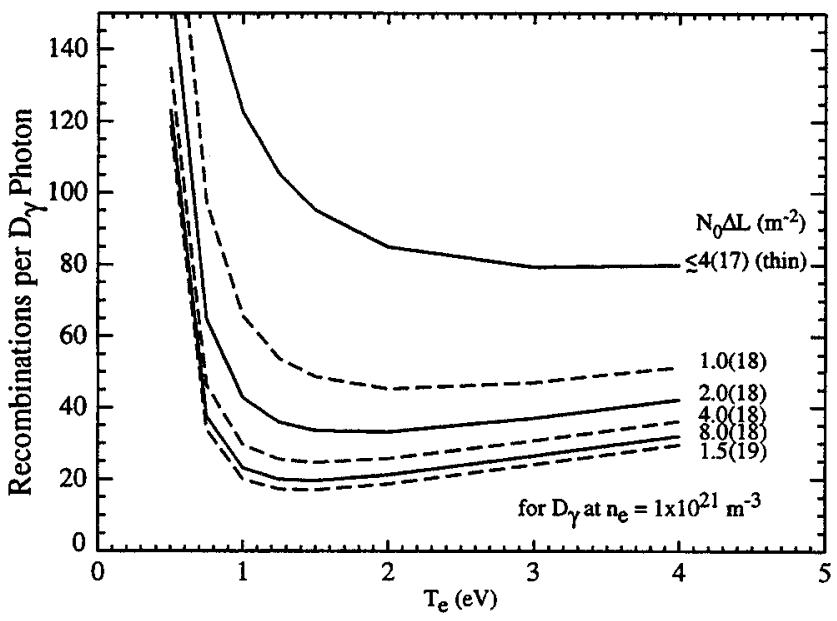

Figure 4: The number of recombinations per $D \gamma$ photon calculated for a recombining plasma with different magnitudes of Lyman line trapping. The $T_{e}$ dependence is relatively weak for $T_{e} \gtrsim \sim 0.8 \mathrm{eV}$ in all cases. The top solid curve is for the optically thin case, applicable for $N_{0} \Delta L \lesssim 4 \times 10^{17} \mathrm{~m}^{-2}$. The other curves show the effects of trapping on the recombination rates. The trapping depends on the number of absorbers along the line of sight, $N_{0} \Delta L$. Each curve is labeled by this quantity $\left(1.0(18)\right.$ means $\left.1.0 \times 10^{18}\right)$.

photon has been lost and no net recombination has occurred, since the 'complete' recombination of the original atom has resulted in an ionization of an absorbing atom. We will use the term 'trapped' to denote those circumstances where there is a net loss of photons due to the combined influence of absorption and collisional processes. The term absorption will be used in its usual sense, not necessarily meaning the final loss of a photon. Trapping and radiation transfer is a very complex problem in which diffusion both in space and wavelength occurs. Here we wish only to define our use of the words "trapped" and "absorption", and consider their influence on the recombination process.

The importance of photon trapping has been considered in Refs. [26,28,18,29]. The primary effect on the ionization/recombination balance is to lower the temperature at which they are equal (for fixed $n_{e}$ and $N_{0}$ ). Experimental observations of trapping of $\mathrm{Ly}_{\beta}$ in the JET divertor plasma have been reported[30]. The radiation transport of Lyman series emission under tokamak-divertor-plasma conditions has been modeled in Ref. [28,31]. The modelling problem is difficult because trapping makes the description of the plasma non-local and non-linear. One experimental 
consequence of trapping is that the measured brightness depends on the density of absorbers between the emitters and the instrument and is, therefore, view dependent.

Because of its importance to the determination of the amount of recombination occurring in these Alcator C-Mod plasmas, the trapping of $\mathrm{Ly}_{\alpha, \beta}$ has been investigated experimentally. The brightnesses of $\mathrm{Ly}_{\beta}$ and $\mathrm{D}_{\alpha}$ were measured by VUV and visible instruments whose views were co-aligned along the "inner nose view". If $\mathrm{Ly}_{\beta}$ is optically thin, the brightnesses of these lines will be in the ratio of their spontaneous emission coefficients [32], i.e.

$$
B_{L y_{\beta}}=B_{D_{\alpha}} \frac{A_{3 \rightarrow 1}}{A_{3 \rightarrow 2}}
$$

where the brightnesses are in units of photons/s/sr/unit-area. An example of a constant ratio being maintained between $\mathrm{Ly}_{\beta}$ and $\mathrm{D}_{\alpha}$ is shown in Fig. 5(a)[33]. However, if $\mathrm{Ly}_{\beta}$ is trapped, its brightness will decrease relative to that of $\mathrm{D}_{\alpha}$. An example of $\mathrm{Ly}_{\beta}$ trapping is shown in Fig. 5(b), where at higher $\mathrm{D}_{\alpha}$ brightness, $\mathrm{Ly}_{\beta}$ falls below the value given on the RHS of Eq. 2. As evidence that this is in no way an instrumental effect, the time history of $\mathrm{Ly}_{\gamma}$, which is measured in the same spectrum as $\mathrm{Ly}_{\beta}$, and which should not be trapped in these plasmas, is compared in Fig. 5(c) to the same $\mathrm{D}_{\alpha}$ time history. $\mathrm{Ly}_{\gamma}$ tracks $\mathrm{D}_{\alpha}$ where $\mathrm{Ly}_{\beta}$ does not.

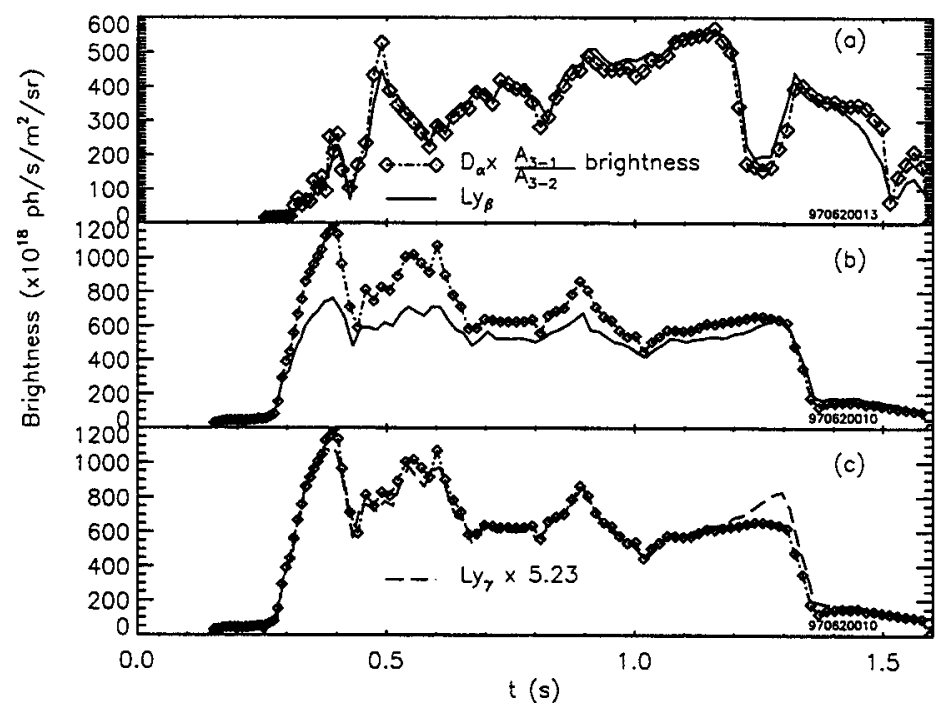

Figure 5: (a) Time histories of the Ly $y_{\beta}$ brightness compared to that predicted by the $D_{\alpha}$ brightness measured with essentially the same view for a discharge where there was no trapping of $L y_{\beta}$. (b) Same as (a), but where some trapping is evident. (c) The time history of $L y_{\gamma}$ for the same discharge as (b), indicating $L y_{\gamma}$ and $D_{\alpha}$ stay in a constant ratio, unlike $L y_{\beta}$ and $D_{\alpha}$. 
The systematic trend of increased trapping of $\mathrm{Ly}_{\beta}$ with increasing $\mathrm{D}_{\alpha}$ emission is shown in Fig. 6(a). At the highest $\mathrm{D}_{\alpha}$ brightnesses, the transmission of $\mathrm{Ly}_{\beta}, \mathrm{T}_{L y_{\beta}}$ - defined as of the ratio of the LHS to the RHS of Eq. 2, has decreased to $\sim 50 \%$. This transmitted fraction may be used to estimate the atomic density in the emitting region. If a $\mathrm{Ly}_{\beta}$ photon is absorbed, there is a high probability that it will not be re-radiated, i.e. it will be trapped. (Note that this is not the case for $\mathrm{Ly}_{\alpha}$, because the radiative rate from $n=2$ is large compared to the collisional rates.) Thus from very simple considerations, only the nearest $\lambda_{m f p}$ of the emitting region will pass $\mathrm{Ly}_{\beta}$ photons and, for transmissions $<1$, the $\mathrm{Ly}_{\beta}$ transmission is

$$
T_{L_{y_{\beta}}} \approx \frac{\lambda_{m f p}}{\Delta L}
$$

The dimension of the emitting region along the line of sight, $\Delta \mathrm{L}$, has been inferred to be $\sim 0.02 \mathrm{~m}$, as described in Sect. III, using optically thin lines and the continuum emission around $\lambda_{\text {edge }}$. Evaluating $\lambda_{m f p}^{L y_{\beta}}$ at the minimum measured transmission of 0.5 implies that the average atomic density in the region is $\sim 1-2 \times 10^{20} \mathrm{~m}^{-3}$. The absorption mean-free-paths of $\mathrm{Ly}_{\alpha}$ and $\mathrm{Ly}_{\gamma}$, evaluated for an atomic density of $2 \times 10^{20}$ $\mathrm{m}^{-3}$, are 0.001 and $0.018 \mathrm{~m}$ respectively. This indicates that $\mathrm{Ly}_{\alpha}$ must be absorbed and re-emitted many times in order to escape, while $\mathrm{Ly}_{\gamma}$ should escape without absorption (consistent with the observation shown in Fig. 5(c)). In order to deal with absorption and trapping in a more quantitative way, the CR code CRAMD has been modified. The transport of photons is considered in a diffusive approximation which results in the reduction of the the spontaneous emission coefficients, $A_{n \rightarrow 1}$, by a factor of $\mathrm{C} /(1+\mathrm{C})$, where $\mathrm{C}=0.33 \times\left(\lambda_{m f p} / \Delta L\right)^{2}$. (Note that these relatively simple modifications account for photon diffusion out of a uniform volume, but do not account for diffusion through the line width profile.) The predictions of the code are in good agreement with the conclusions of the simple argument above using Eq. 3, i.e. at $\mathrm{T}_{L y_{\beta}}=0.5, \mathrm{~N}_{0} \Delta L \sim 3 \times 10^{18} \mathrm{~m}^{-2}$ (for $1 \mathrm{eV}$ atoms in a $1 \mathrm{eV}, 10^{21} \mathrm{~m}^{-3}$ plasma), $\mathrm{T}_{L y_{\gamma}} \approx 1$, and $\mathrm{L}_{\alpha}$ is strongly trapped.

A similar experimental investigation of the trapping of $\mathrm{Ly}_{\alpha}$ cannot be done since there is no other radiative decay branch from $n=2$. However, the predicted strong trapping of $\mathrm{Ly}_{\alpha}$ is consistent with the observation shown in Fig. 6(b), where the 


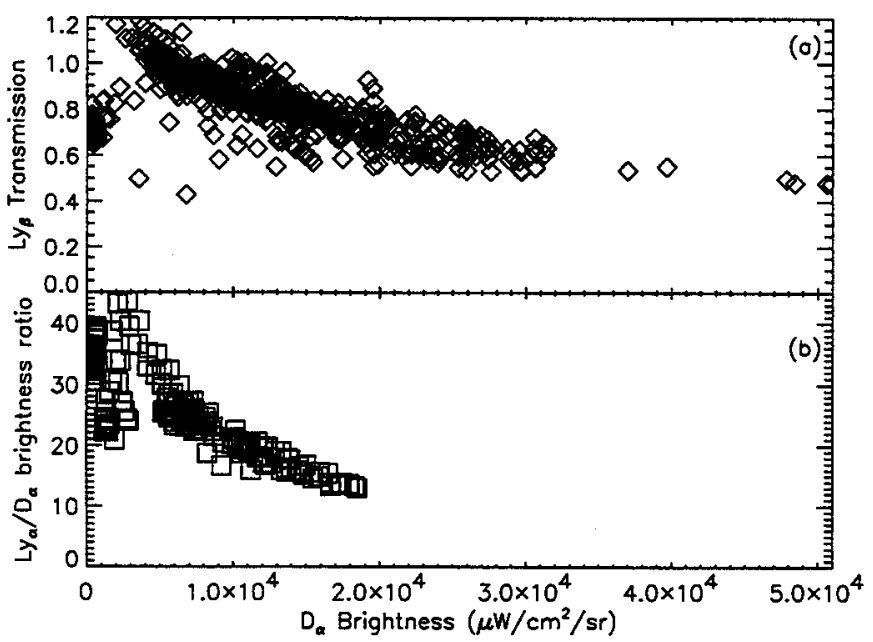

Figure 6: (a) The scaling of the transmitted fraction of $L y_{\beta}$ vs. the $D_{\alpha}$ brightness. (b) The scaling of the ratio of $L y_{\alpha}$ to $D_{\alpha}$ brightnesses (measured in photons $/ \mathrm{s} / \mathrm{cm}^{2} / \mathrm{sr}$ ) vs. the $D_{\alpha}$ brightness.

$\mathrm{Ly}_{\alpha} / \mathrm{D}_{\alpha}$ brightness is plotted vs the $\mathrm{D}_{\alpha}$ brightness. This plot indicates probable $\mathrm{Ly}_{\alpha}$ trapping, since the brightness ratio is decreasing faster than the $\mathrm{Ly}_{\beta}$ transmission.

CRAMD, modified to account for absorption and trapping, has been used to calculate 'recombinations per photon' in those cases where Lyman series lines are trapped. The 'recombinations per photon' curves for a number of values of $N_{0} \Delta L$ are shown in Fig. 4. The curve for $N_{0} \Delta L=4 \times 10^{18} \mathrm{~m}^{-2}$ is close to the extreme case implied by the measurements shown in Fig. 6, i.e. for $T_{L y_{\beta}} \sim 0.5$. As expected, the values are reduced from the the optically thin case, typically by a factor of $\sim 5$. This is due to the decrease in the net recombination rate which occurs when such a large fraction of the $\mathrm{Ly}_{\alpha}$ radiation does not escape. Note that neither the net rate nor the 'recombinations per photon' decreases significantly beyond the $\mathrm{N}_{0} \Delta L=4 \times 10^{18}$ $\mathrm{m}^{-2}$ curve, since most of the $\mathrm{Ly}_{\alpha}$ emission is already trapped. As in the optically thin case, the dependence on $\mathrm{T}_{e}$ is weak above $\sim 0.8 \mathrm{eV}$, and the dependence upon $\mathrm{n}_{e}$ (not shown) is weak as well. For example, at $\mathrm{N}_{0} \Delta L=8 \times 10^{18} \mathrm{~m}^{-2}$ and $1 \mathrm{eV}$ the 'recombinations per $\mathrm{D}_{\gamma}$ photon' decreases by $23 \%$ if the electron density decreases from 1 to $0.5 \times 10^{21} \mathrm{~m}^{-3}$.

\section{RECOMBINATION RESULTS AND DISCUSSION}


The analysis techniques developed in Sections III and IV have been applied to estimate the ion sink due to 3-body and radiative recombination in Alcator C-Mod. The importance of this sink has been evaluated by comparing it to the rate at which ions are lost to the divertor plates, as measured by sets of probes embedded in them[1]. The spatial distribution and magnitude of the recombination have been measured using the spatially resolved views in the "inner and outer divertor fans" which view the strike points. After finding those lines whose upper levels are populated primarily by recombination, the recombination rate within each field-of-view has been found from the 'recombination per photon' curves. The magnitude of the trapping will determine which 'recombination per photon' curve is appropriate. This can be estimated based on the scaling shown in Fig. $6(\mathrm{a})$. The maximum $\mathrm{D}_{\alpha}$ brightness at this time was $\sim 8 \times 10^{4} \mu \mathrm{W} / \mathrm{cm}^{2} /$ ster (along a chord viewing the inner strike point). $\mathrm{D}^{0}$ brightnesses along the outer divertor views were typically a factor of 5 lower than those of the inner divertor, and it is therefore likely that both $\mathrm{Ly}_{\alpha}$ and $\mathrm{Ly}_{\beta}$ are strongly trapped along the inner divertor views and at least $\mathrm{Ly}_{\alpha}$ is trapped for the outer views. Thus the 'recombinations per photon' curves corresponding to $\mathrm{N}_{0} \Delta L=8 \times 10^{18} \mathrm{~m}^{-2}$ were used conservatively for both. The spatial distribution of recombination is shown in Fig. 7 at a time when the plasma was detached from the inner divertor plate, but was attached at the outer plate.

As seen in Fig. 7, the deduced recombination rates, are peaked for chords viewing near the strike points on the respective plates. Radial profiles of $D_{\alpha}$ emission from the "top divertor view" show that most of the emission from the divertor is from the region close to the inner divertor. For this reason, it is assumed that the emission measured by the chords in the "inner divertor fan" is coming from close to the inner divertor, and is therefore a measure of the recombination rate at the inner divertor. The spatially integrated recombination rate in the detached region in front of the inner divertor plate is $\sim 60 \%$ of the total ion current to the inner plate $\left(\sim 1 \times 10^{22} \mathrm{~s}^{-1}\right)$ and is thus a significant fraction $(\sim 40 \%)$ of the total ion sink. The recombination rate at the inner divertor is peaked near the inner strike point, and drops sharply below it. This also implies that the recombination is occurring near the plate. At other times, the recombination at the inner plate is observed to move away from the strike point, up toward or beyond the inner divertor "nose". 


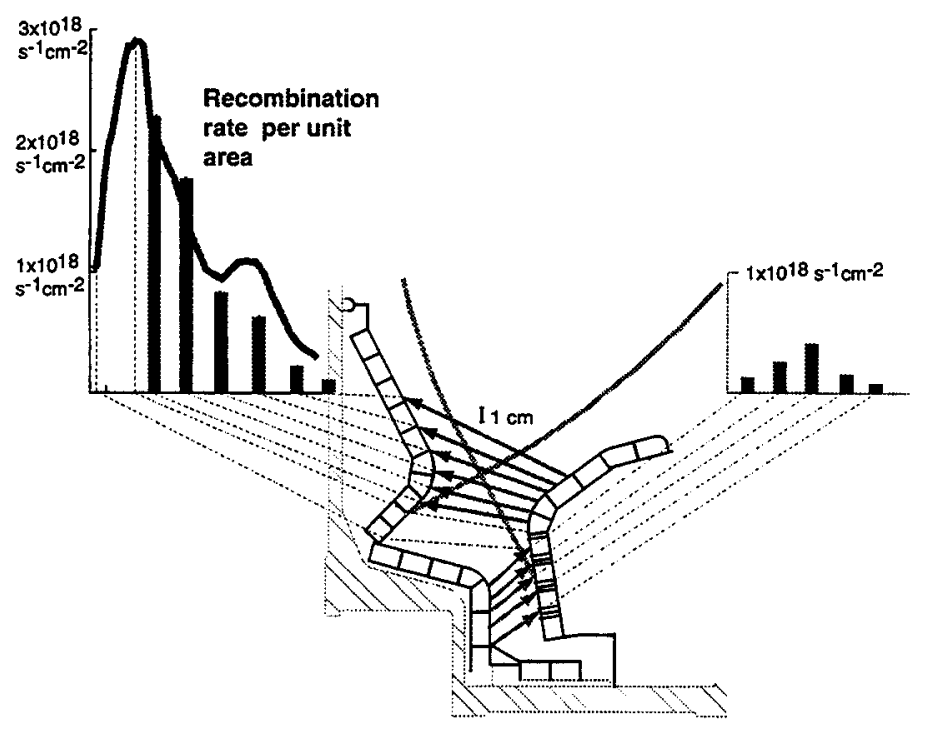

Figure 7: The recombination rate per unit area determined for different viewed areas of the inner and outer divertor plates. The bars on the inner divertor profile are derived from Balmer series spectra, while the smooth profile uses the $D_{\alpha}$ array measurements. At this time the inner divertor plate is detached, while the outer is not. Also shown are the viewing chords, the LCFS, and the strike points.

For the attached outer plate, the ion loss to the plate is $\sim 4 \times 10^{22} \mathrm{~s}^{-1}$, half of which is collected below the outer "nose". The recombination rate occurring within the viewed region $\left(\sim 7 \times 10^{20} \mathrm{~s}^{-1}\right)$ is small in comparison. In this case the rate is also peaked near the strike point. The points to be stressed by this example are:

- The recombination as a fraction of the total ion sink is much greater for the case where the divertor is detached.

- Under detached conditions that fraction can be large.

In order to examine the recombination rate under dynamic conditions, the time histories of the recombination rate at the inner detached plate and the ion current to that plate for another discharge have been evaluated and are shown in Fig. 8. The "inner divertor view" fan was used for the spectroscopic measurements, and the 'recombinations per photon' curve corresponding to the strongly trapped case was used, since this was implied by the magnitude of the $D_{\alpha}$ brightnesses. It is evident from the comparisons shown in Figs. 8(b) and (c) that the recombination sink can be greater than the ion current sink even under conditions when $\operatorname{Ly}_{\alpha}$ and $\mathrm{Ly}_{\beta}$ are trapped. During this discharge there are four distinct periods of $\mathrm{H}$-mode core 


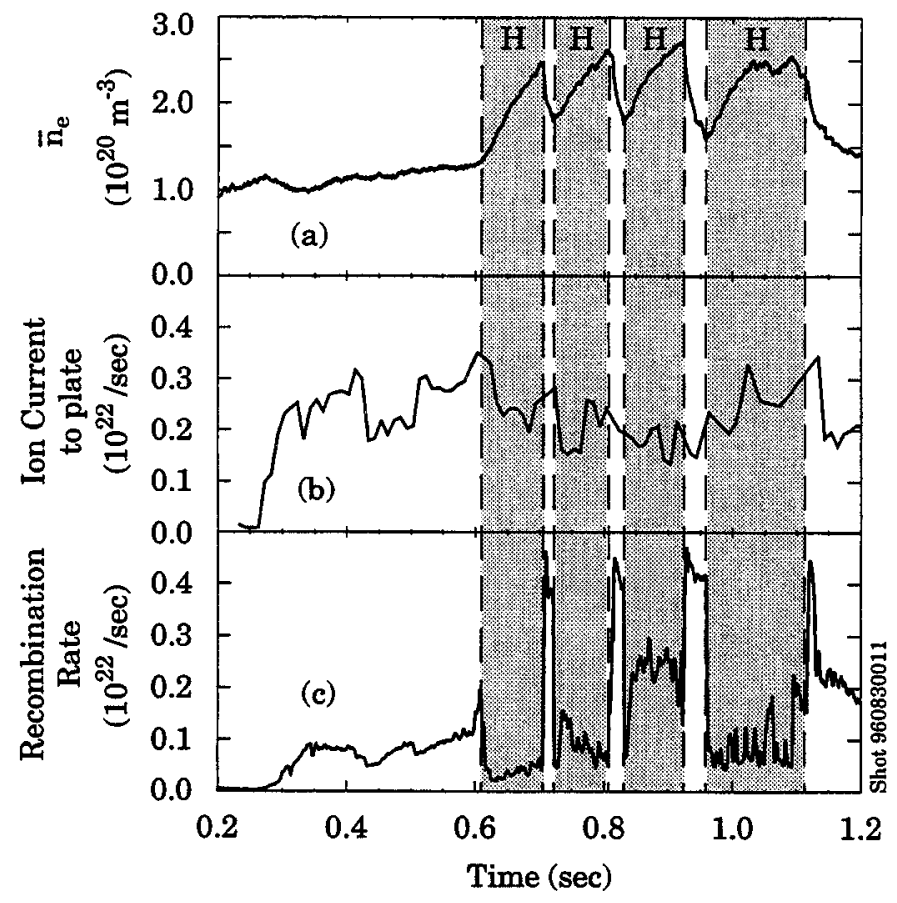

Figure 8: Time histories of (a) main plasma density with designations for those time periods when there is $H$-mode core confinement, (b) the total ion current to the detached inner divertor plate, and (c) the recombination rate at the inner plate, evaluated using the recombination-per-photon formalism, assuming $L y_{\alpha, \beta}$ are trapped. 
confinement. They are separated by short periods of L-mode core confinement. The recombination sink is strongly modulated by the core confinement mode, probably as a result of changing edge conditions away from the divertor. The ion current is much less well correlated to the confinement mode, but generally behaves in the same way, i.e. the ion sinks decrease at the $\mathrm{L}-\mathrm{H}$ transition and increase at the $\mathrm{H}$ $\mathrm{L}$ transition. Even though the recombination rate decreases at the L-H transition, during $\mathrm{H}$-Mode it can be approximately the same magnitude as the ion current, as seen during the third $\mathrm{H}$-mode period. In addition, the large variations in magnitude of both the recombination sink and the total ion sink imply that the ionization source is changing.

The measured magnitude of the recombination is in rough agreement with that predicted for detached Alcator C-Mod discharges as modelled by the 2-D plasma code UEDGE[34] coupled to a 2-D Navier-Stokes neutral code[35]. UEDGE predicts a recombination sink that is $\sim 0.3$ of the ionization source. However, these UEDGE predictions do not account for radiation trapping. Regarding the spatial distribution of the recombination, the UEDGE modelling shows that the recombination is peaked in the regions just above the strike points, in qualitative agreement with the experiment, but does not exhibit the wide variety of distributions seen in the experiment.

\section{MOLECULAR ACTIVATED RECOMBINATION}

Experimental investigations seeking to identify and measure MAR in tokamak divertors are of interest because MAR has been predicted to be an important pathway for volume recombination[8]. We have modelled measured Lyman series spectra emitted from a relatively low density discharge for which the inner divertor was detached. If the modelling does not include MAR effects, the model predictions do not agree with the measurements within the experimental uncertainties. When MAR is included there is much better agreement with the experiment. This is strong evidence that MAR is occurring in the divertor.

The measurements of the Lyman series lines ( $\mathrm{Ly}_{\alpha}$ through $\left.\mathrm{Ly}_{\epsilon}\right)$ were made along the "inner nose view". Both the $\mathrm{Ly}_{\beta}$ and $\mathrm{D}_{\alpha}$ brightnesses were quite low, and $\mathrm{Ly}_{\beta}$ was not trapped. The measured spectra show the Lyman series lines in intensity ratios of $433\left(\mathrm{Ly}_{\alpha}\right)$ to $40\left(\mathrm{Ly}_{\beta}\right)$ to $7.4\left(\mathrm{Ly}_{\gamma}\right)$ to $2.1\left(\mathrm{Ly}_{\delta}\right)$ to $1\left(\mathrm{Ly}_{\epsilon}\right)$. 


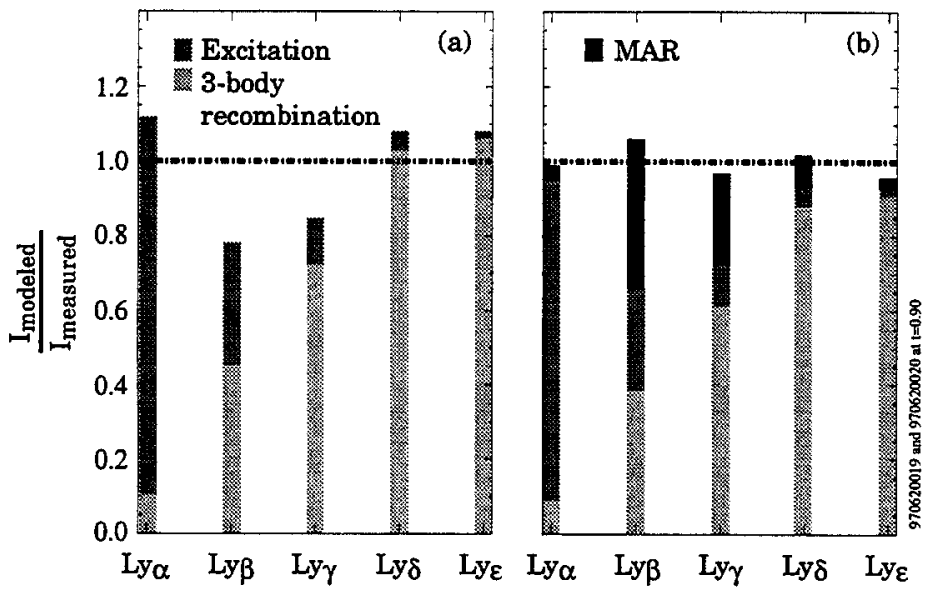

Figure 9: The ratios of the modelled and measured brightnesses of the Lyman series lines for two cases: (a) the best fit in the case in which MAR was not included in the model, and (b) the best fit when MAR was included. The bars give the relative contributions of 3-body recombination (light shade), excitation (medium shade), and MAR (dark shade) to populating these lines.

In an initial attempt to model these intensity ratios, it was assumed that the emission was from a single isothermal region. As in the Balmer series spectra, the ratios for the $n \gtrsim 5$ transitions are easily modelled as coming from a 'cold' ( $\mathrm{T}_{e}^{\text {cold }} \approx 1$ $\mathrm{eV}$ ) region where 3-body recombination populates the upper levels. (This is obtained for $\mathrm{n}_{e}^{\text {cold }}=3.8 \times 10^{20} \mathrm{~m}^{-3}$, and $\Delta L^{\text {cold }}=0.02 \mathrm{~m}$.) However, a recombining spectrum cannot account for the $\mathrm{Ly}_{\alpha, \beta, \gamma}$ emission which is much greater than predicted by recombination alone. To raise the predicted $\mathrm{Ly}_{\beta}$ brightness to that measured by modelling excitation from this 'cold' plasma requires an atomic density $\approx 80$ times greater than $\mathrm{n}_{e}^{\text {cold }}$. As a result, a hotter $(\sim 2-5 \mathrm{eV})$ emission region within the field-ofview has also been postulated. Using CRAMD and including possible trapping effects (within the constraint that $\mathrm{Ly}_{\beta}$ was not trapped), the spectrum has been modelled considering emission by excitation and 3-body recombination from two such regions. The best fit to the measurements under this two region assumption is shown in Fig. 9(a). The relative contributions for excitation and 3-body recombination are shown as well. (For this case $\mathrm{n}_{e}^{\text {cold }}$ was taken to be $4.0 \times 10^{20} \mathrm{~m}^{-3}$ which over predicts the $\mathrm{Ly}_{\delta, \epsilon}$ intensities in order to maximize the quality the overall fit. The parameters for the 'hot' region for this fit were $\mathrm{T}_{e}^{h o t}=4 \mathrm{eV}, \mathrm{N}_{0}^{h o t} \times \mathrm{n}_{e}^{h o t}=0.13 \times 10^{40} \mathrm{~m}^{-6}$, and $\Delta L^{h o t}=0.02$ 
m.) It is clear that predictions considering excitation and 3-body recombination alone over predict the $\mathrm{Ly}_{\alpha}, \mathrm{Ly} y_{\delta}$, and $\mathrm{Ly}_{\epsilon}$ intensities relative to those for $\mathrm{Ly}_{\beta}$ and $\mathrm{Ly}_{\gamma}$. They do so to an extent outside the experimental uncertainties for the line ratios.

Inclusion of MAR in the model considerably improves the agreement between the model predictions and the measurements, as is shown in Fig. 9(b). MAR leaves atoms in the $n=2-4$ excited states, and therefore makes up for the deficits in the $\operatorname{Ly}_{\beta}$ and $\mathrm{Ly}_{\gamma}$ intensities predicted without it. CRAMD calculates the distribution among the excited states resulting from $\mathrm{MAR}[20]$ and accounts for any $\mathrm{Ly}_{\alpha}$ trapping. Only the molecular density, $\mathrm{N}_{D_{2}}$, need be specified, and the contributions of MAR to all of the levels are determined. MAR will be significant only in the 'cold' region. Taking the conditions for the cold region similar to those used for the fit of Fig. 9(a), and including the contributions to the levels populations due to MAR with $N_{D_{2}}=0.05 \times n_{e}^{\text {cold }}$, still requires some contribution to $\mathrm{Ly}_{\alpha}$ and $\mathrm{Ly}_{\beta}$ from a hot region. $\left(\mathrm{N}_{0}^{\text {hot }} \times n_{e}^{\text {hot }}=0.11 \times 10^{40}\right.$ $\mathrm{m}^{-6}$.) The relative contributions from each process are shown in the figure. Under these conditions the recombination rate due to MAR is $\sim 1.5$ times the rate due to 3 -body and radiative recombination. To compare the recombination sink with the measured ion current to the inner divertor plate, we assume the emission is uniform throughout a toroidally symmetric divertor volume of $0.02 \mathrm{~m}$ radial width and $0.1 \mathrm{~m}$ height and calculate the total recombination rate to be $\sim 2.7 \times 10^{21} \mathrm{~s}^{-1}$. The measured ion current to the inner plate at this time is $\sim 5 \times 10^{21} \mathrm{~s}^{-1}$, and the total predicted ionization rate from the hot region, which must supply these sinks, is calculated to be $\sim 1 \times 10^{22} \mathrm{~s}^{-1}$. Thus the plasma sources and sinks are approximately consistent.

\section{CONCLUSIONS}

Temperatures and densities in Alcator C-Mod detached divertor plasmas are measured to be $\sim 1 \mathrm{eV}$ and $\lesssim 1.5 \times 10^{21} \mathrm{~m}^{-3}$. Under these conditions volume recombination is a significant sink for ions and is in some instances a greater sink for ions than ion flow to the plate. This has been measured by examining Balmer series spectra and finding lines whose intensities are related to the 3-body recombination rate. This relationship has been expressed in the 'recombinations per photon' curves which are rather weak functions of $\mathrm{T}_{e}$ (above $\sim 0.8 \mathrm{eV}$ ) and $n_{e}$.

The recombination rates in these low temperature conditions are dependent on the absorption and trapping of the Lyman lines, primarily $\mathrm{Ly}_{\alpha}$. It is shown that 
$\mathrm{Ly}_{\alpha}$ is strongly trapped in most cases in C-Mod in the recombination regions. This reduces the 3 -body recombination rates by up to a factor of $\sim 5$.

The magnitude of the recombination rate is found to be greater when the plasma is detached. Examination of the magnitude and time histories of the ion sinks (flow to the plates and volume recombination) shows that both the recombination and the total sink are changed by the confinement mode of the core plasma.

Evidence for MAR is based on modelling of Lyman series spectra. The inferred recombination rate from this process is roughly the same magnitude as the 3-body recombination rate.

\section{ACKNOWLEDGEMENTS}

This work is supported by the U.S. Department of Energy under the contract \# DE-AC02-78ET51013 and under the grant \#DE-FG02-910ER-54109. The authors acknowledge help from the entire Alcator C-Mod physics staff and the excellent operational support by the engineering and technical staff. One of us (JLT) thanks especially I.H. Hutchinson, E.S. Marmar, J.A. Goetz, G.M. McCracken, C.S. Pitcher, and J.E. Rice for very helpful discussions.

\section{REFERENCES}

[1] B. LaBombard, J.A. Goetz, C. Kurz, D. Jablonski, B. Lipschultz, G.M. McCracken, A. Niemczewski, R. Boivin, F. Bombarda, P. Bonoli, C. Christenson, S. Fairfax, C. Fiore, D. Garnier, M. Graf, S. Golovato, R. Granetz, M. Greenwald, S. Horne, A. Hubbard, I.H. Hutchinson, J. Irby, J. Kesner, T. Luke, E. Marmar, M. May, P. O'Shea, M. Porkolab, J. Reardon, J. Rice, J. Schacter, J. Snipes, P. Stek, Y. Takase, J.L. Terry, G. Tinios, R. Watterson, B. Welch, and S. Wolfe. Phys. Plasmas, 2: 2242, 1995.

[2] S. L. Allen, D. N. Hill, T. N. Carlstrom, D.G. Nilson, T.W. Petrie, A.W. Leonard, D. Ryutov, G.D. Porter, R. Maingi, M.R. Wade, R.H. Cohen, W.M. Nevins, M.E. Fenstermacher, R.D. Wood, C.J. Lasnier, W.P. West, and M.D. Brown. J. Nucl. Mater., 241-243:595-601, 1997.

[3] G.F. Matthews. J. Nucl. Mater., 220-222: 104, 1995.

[4] A. Loarte. J. Nucl. Mater., 241-243:118-134, 1997.

[5] F. Wising, D.A. Knoll, S.I. Krasheninnikov, T.D. Rognlien, and D.J. Sigmar. Contrib. Plasma Phys., 36:136, 1996.

[6] G.D. Porter, S.L. Allen, M. Brown, M.E. Fenstermacher, D.N. Hill, R. Jong, A.W. Leonard, D.G. Nilson, M.E. Resnik, T.D. Rognlien, G.R. Smith, and the DIIID Team. Phys. Plasmas, 3:1967, 1996.

[7] K. Borrass, D. Coster, D. Reiter, and R. Schneider. J. Nucl. Mater., 241-243:250-254, 1997. 
[8] S.I. Krasheninnikov, A. Yu. Pigarov, D.A. Knoll, B. LaBombard, B. Lipschultz, D.J. Sigmar T. K. Soboleva, J. L. Terry, and F. Wising. Phys. Plasmas, 4:1638, 1997.

[9] M.E. Fenstermacher, S.L. Allen, N.H. Brooks, D.A. Buchenauer, T.N. Carlstrom, J.W. Cuthbertson, E.J. Doyle, T.E. Evans, P.-M. Garbet, R.W. Harvey, D.N. Hill, A.W. Hyatt, R.C. Isler, G. Jackson, R.A. James, R. Jong, C.C Klepper, C.J. Lasnier, A.W. Leonard, M.A. Mahdavi, R. Maingi, W.H. Meyer, R.A. Moyer, D.G. Nilson, T.W. Petrie, G.D. Porter, T.L. Rhodes, M.J. Schaffer, R.D. Stambaugh, D.M. Thomas, S. Tugarinov, M.R. Wade, J.G. Watkins, W.P. West, D.G. Whyte, , and R.D. Wood. Phys. Plasmas, 4:1761, 1997.

[10] D. Lumma, J.L. Terry, and B. Lipschultz. Phys. Plasmas, 4:2555, 1997.

[11] J.L. Terry, B. Lipschultz, B. LaBombard, and D. Pappas. Proc. of the 24th European Phys. Soc. Conf. on Contr. Fus. and Plasma Phys. - Vol 21 A - PartII. Page 573, European Physical Society, Petit-Lancy, 1997.

[12] B. Napiontek, U. Wenzel, K. Behringer, D. Coster, R. Schneider, T. Thomas, M. Weinlich, and the ASDEX Upgrade Team. Proc. of the 24th European Phys. Soc. Conf. on Contr. Fus. and Plasma Phys. - Vol 21A - PartIV. Page 1413, European Physical Society, Petit-Lancy, 1997.

[13] R. Isler, G.R. McKee, N.H. Brooks, W.P. West, M.E. Fenstermacher, and R.W. Wood. Plasma Physics, 4: 2989, 1997.

[14] G.M. McCracken, M.F. Stamp, R.D. Monk, A. Meigs, J. Lingerat, R. Prentice, A. Starling, and R. Smith. Evidence for Volume Recombination in JET Detached Divertor Plasmas. submitted to Nucl. Fus.

[15] G.S. Chiu and S.A. Cohen. Phys. Rev. Lett., 76:1248, 1996.

[16] N. Ezumi, N. Ohno, Y. Uesugi, J. Park, S. Watanabe, S.A. Cohen, S.I. Krasheninnikov, A. Yu. Pigarov, M. Takagi, and S. Takamura. Proc. of the 24th European Phys. Soc. Conf. on Contr. Fus. and Plasma Phys. - Vol 21A - PartIII. Page 1225, European Physical Society, Petit-Lancy, 1997.

[17] R. K. Janev, D.E. Post, W.D. Langer, K. Evans, D.B. Heifetz, and J.C. Weisheit. J. Nucl. Mater., 121:10, 1984.

[18] D.E. Post. J. Nucl. Mater., 220-222: 143, 1995.

[19] S.I. Krashenninikov, A.Yu. Pigarov, and D.J. Sigmar. Phys. Lett., 214:285, 1996.

[20] A.Yu. Pigarov and S.I. Krashenninikov. Phys. Lett. A, 222:251, 1996.

[21] J. L. Terry, J. A. Snipes, and C. Kurz. Rev. Sci. Instrum., 66: 555, 1995.

[22] H. Ohkawa. Determination of the Spectral Sensitivity of a VUV, Grazing Incidence Spectrograph. M.I.T. M.S. Thesis, PSFC Report PSFC/RR-97-11 (1997).

[23] I. H. Hutchinson, R. Boivin, F. Bombarda, P. Bonoli, S. Fairfax, C. Fiore, J. Goetz, S. Golovato, R. Granetz, M. Greenwald, S. Horne, A. Hubbard, J. Irby, B. LaBombard, B. Lipschultz, E. Marmar, G. McCracken, M. Porkolab, J. Rice, J. Snipes, Y. Takase, J. Terry, S. Wolfe, C. Christenson, D. Garnier, M. Graf, T. Hsu, T. Luke, M. May, A. Niemczewski, G. Tinios, J. Schachter, and J. Urbahn. Phys. Plasmas, 1: $1511,1994$. 
[24] B. Lipschultz, J.A. Goetz, B. LaBombard, G.M. McCracken, H. Ohkawa, Y. Takase, and J.L. Terry. I. Nucl. Mater., 241-243:771, 1997.

[25] A. Yu. Pigarov, J.L. Terry, and B. Lipschultz. Proc. of the 24th European Phys. Soc. Conf. on Contr. Fus. and Plasma Phys. - Vol 21A - PartII. Page 577, European Physical Society, Petit-Lancy, 1997.

[26] L. C. Johnson and E. Hinnov. J. Quant. Spectrosc. Radiat. Transfer, 13: 333 - 358, 1973.

[27] T. Fujimoto, S. Miyachi, and K. Sawada. Nucl. Fus., 28: 1255, 1988.

[28] S.I. Krasheninnikov and A. Yu. Pigarov. Contrib. Plasma Phys., 28:443, 1988.

[29] K. Behringer. private communication.

[30] T. Lovegrove, L.D. Horton, P.J. Storey, R.W.T. Konig, J. Lingerat, A.C. Maas, C.F. Maggi, R.D. Monk, M.F. Stamp, and A. Tabasso. Proc. of the 22th European Phys. Soc. Conf. on Contr. Fus. and Plasma Phys. - Vol 19C - PartIII. Page 301. European Physical Society, Petit-Lancy, 1995.

[31] A.S. Wan, H.E. Dalhed, H.A. Scott, D.E. Post, and T.D. Rognlien. J. Nucl. Mater., 220-222:1102, 1995.

[32] This is true as long as the sub levels of $n=3$ are statistically populated. Statistical population of the sub-levels occurs very quickly and obtains in these plasmas.

[33] Actually the VUV spectrometer's sensitivity has been calibrated by this 'branching ratio' technique on lower density discharges like the one shown in Fig. 5(a), when both lines are still optically thin. The systematic change with $\mathrm{D}_{\alpha}$ brightness indicative of $\mathrm{Ly}_{\beta}$ trapping is shown in Fig. 6 .

[34] T.D. Rognlien, J.L. Milovich, M.E. Resnik, and G.D. Porter. J. Nucl. Mater., 196198: 347, 1992.

[35] D.A. Knoll, R.P. McHugh, S.I. Krasheninnikov, and D.J. Sigmar. Phys. Plasmas, 3: 293, 1996. 